\title{
A CONVERGENCE THEOREM FOR CERTAIN LAGRANGE INTERPOLATION POLYNOMIALS
}

M. S. WEBSTER

In the Lagrange interpolation polynomial $L_{n}[f ; \theta]$ where

$$
\begin{aligned}
L_{n}[f ; \theta] & \equiv \sum_{k=1}^{n} f\left(x_{k}\right) l_{k}[\theta], \\
l_{k}[\theta] & \equiv l_{k}^{(n)}[\theta] \equiv l_{k}(x) \equiv \frac{\phi_{n}(x)}{\phi_{n}^{\prime}\left(x_{k}\right)\left(x-x_{k}\right)}, \\
\phi_{n}(x) & \equiv \prod_{k=1}^{n}\left(x-x_{k}\right), \\
x=\cos \theta ; & -1<x_{k}<1 ; k=1,2, \cdots, n ; n=1,2, \cdots,
\end{aligned}
$$

and $f(x)$ is a continuous function defined in $(-1,1)$, we suppose that

$$
x_{k} \equiv x_{k}^{(n)}=\cos \theta_{k}=\cos k \pi /(n+1) .
$$

Then $[1],{ }^{1}$ we have

$$
\begin{aligned}
\phi_{n}(x) & =\frac{\sin (n+1) \theta}{2^{n} \sin \theta}, \\
l_{k}[\theta] & =\frac{(-1)^{k+1} \sin ^{2} \theta_{k} \sin (n+1) \theta}{(n+1) \sin \theta\left(\cos \theta-\cos \theta_{k}\right)} .
\end{aligned}
$$

We introduce the following notations:

$$
\begin{gathered}
t_{n} \equiv t \equiv \theta_{1} / 2 \equiv \pi / 2(n+1), \quad M=\max _{-1 \leqq x \leqq 1}|f(x)|, \\
S_{k}[\theta] \equiv\left\{l_{k}[\theta-t]+l_{k}[\theta+t]\right\} / 2 .
\end{gathered}
$$

We shall prove the following theorem which was suggested by a similar theorem of Grünwald [2].

THEOREM. Let $f(x)$ be a continuous function in the interval $-1 \leqq x \leqq 1$. Then

(5) $\lim _{n \rightarrow \infty}(1 / 2)\left\{L_{n}\left[f ; \theta-t_{n}\right]+L_{n}\left[f ; \theta+t_{n}\right]\right\}=f(\cos \theta), \quad 0<\theta<\pi$, and the convergence is uniform in the interval $0<\alpha \leqq \theta \leqq \pi-\alpha$ ( $\alpha$ arbi-

Presented to the Society, April 18, 1942; received by the editors April 10, 1942.

1 The numbers in brackets refer to the bibliography. 
rary, fixed constant). In general, convergence does not hold for $\theta=0$ or $\theta=\pi$.

We shall prove first that there is a constant $D$ for which

$$
\sum_{k=1}^{n}\left|S_{k}[\theta]\right|<D, \quad 0<\alpha \leqq \theta \leqq \pi-\alpha ; n=1,2, \cdots .
$$

If $\theta \neq \theta_{k} \pm t$, it follows from (3) and (4), by the use of trigonometric addition formulas, that

$$
\begin{aligned}
& S_{k}[\theta]=\frac{(-1)^{k+1} \sin t \sin ^{2} \theta_{k} \cos (n+1) \theta}{4(n+1) \sin (\theta-t) \sin (\theta+t)} \\
& \cdot \frac{\cos \theta \cos \theta_{k}-\cos 2 \theta \cos t}{\sin \left(\theta+\theta_{k}-t\right) / 2 \sin \left(\theta-\theta_{k}-t\right) / 2 \sin \left(\theta+\theta_{k}+t\right) / 2 \sin \left(\theta-\theta_{k}+t\right) / 2} .
\end{aligned}
$$

If $\theta$ is restricted to the interval $0<\alpha \leqq \theta \leqq \pi / 2$, and if $n$ is large so that $t \leqq \alpha / 2$, it is easily seen (assuming $\alpha \leqq \pi / 3$ ) that

$$
\begin{aligned}
& \left|\frac{\sin ^{2} \theta_{k}}{\sin (1 / 2)\left(\theta+\theta_{k}-t\right) \sin (1 / 2)\left(\theta+\theta_{k}+t\right)}\right|<4, \\
& \left|S_{k}[\theta]\right|<\frac{\pi^{3} C}{(n+1)^{2}\left|\theta-\theta_{k}-t\right| \cdot\left|\theta-\theta_{k}+t\right|}, \\
& |\csc (\theta-\lambda) \csc (\theta+\lambda)| \leqq \frac{2}{\cos \alpha-\cos 2 \alpha} \equiv C, \\
& \alpha \leqq \theta \leqq \pi / 2,0 \leqq \lambda \leqq \alpha / 2 .
\end{aligned}
$$

For a given $\theta$, there are at most two values of $k$ for which $\left|\theta-\theta_{k}\right|<\pi /(n+1)$. Since [1] $\left|l_{k}(x)\right|<2(-1 \leqq x \leqq 1 ; k=1,2, \cdots, n$; $n=1,2, \cdots)$, from (8) we have

$$
\begin{aligned}
\sum_{k=1}^{n}\left|S_{k}[\theta]\right| & <4+\sum_{1 \leqq k \leqq n,|\theta-\theta k| \geqq \pi /(n+1)}\left|S_{k}[\theta]\right| \\
& <4+\frac{\pi^{3} C}{(n+1)^{2}} \sum_{1 \leqq k \leqq n,|\theta-\theta k| \geqq \pi /(n+1)} \frac{1}{\left|\theta-\theta_{k}-t\right| \cdot\left|\theta-\theta_{k}+t\right|} \\
& <4+\frac{2 \pi^{3} C}{(n+1)^{2}} \sum_{l=1}^{n}\left[\frac{2(n+1)}{\pi}\right]^{2} \frac{1}{(2 l-1)^{2}} \\
& <4+8 \pi C \sum_{m=1}^{\infty} \frac{1}{m^{2}} \equiv D^{\prime}, \quad 0<\alpha \leqq \theta \leqq \pi / 2 ; n \geqq n_{0}(\alpha),
\end{aligned}
$$


because in the summation

$$
\left|\theta-\theta_{k} \pm t\right| \geqq\left|\theta-\theta_{k}\right|-t \geqq l \pi /(n+1)-\pi / 2(n+1), \quad l \geqq 1 .
$$

By continuity, (9) holds for all $\theta$ in $0<\alpha \leqq \theta \leqq \pi / 2$. Since (9) is valid for $n$ sufficiently large, there exists a $D$ for which (6) is valid for all $n$ if $0<\alpha \leqq \theta \leqq \pi / 2$.

Since $\theta_{k}=\pi-\theta_{n-k+1}$, it is found that

$$
\left.l_{k}[\theta-t]=l_{n-k+1}\left[\theta^{\prime}+t\right] \text { and } l_{k}[\theta+t]=l_{n-k+} \theta^{\prime}-t\right]
$$

where $\theta^{\prime}=\pi-\theta$. It follows that

$$
S_{k}[\theta]=S_{n-k+1}\left[\theta^{\prime}\right], \quad \sum_{k=1}^{n}\left|S_{k}\left[\theta^{\prime}\right]\right|<D, \quad \pi / 2 \leqq \theta^{\prime} \leqq \pi-\alpha .
$$

This completes the proof of (6).

From (8), if $\delta(>0)$ is fixed and if $n$ is sufficiently large (so that $t<\delta / 2)$, it is seen that

$$
\begin{aligned}
\sum_{1 \leqq k \leqq n,\left|\theta-\theta_{k}\right|>\delta}\left|S_{k}[\theta]\right| & <\frac{\pi^{3} C}{(n+1)^{2}} \sum_{k=1}^{n} \frac{1}{(\delta-t)^{2}} \\
& <\frac{4 \pi^{3} C n}{\delta^{2}(n+1)^{2}}=O\left(\frac{1}{n}\right) .
\end{aligned}
$$

We are now ready to prove the main part of the theorem. Let $\theta$ be fixed $(0<\alpha \leqq \theta \leqq \pi-\alpha)$ and $\epsilon>0$. It is well known that

$$
\sum_{k=1}^{n} l_{k}(x) \equiv 1, \quad \sum_{k=1}^{n} S_{k}[\theta] \equiv 1 .
$$

Since $f(x)$ is continuous, there exists a $\delta>0$ such that

$$
\left|f(\cos \theta)-f\left(\cos \theta_{k}\right)\right|<\epsilon \text { provided }\left|\theta-\theta_{k}\right| \leqq \delta .
$$

Let

$$
\begin{aligned}
\Delta= & (1 / 2)\left\{L_{n}[f ; \theta-t]+L_{n}[f ; \theta+t]\right\}-f(\cos \theta) \\
= & \sum_{k=1}^{n}\left\{f\left(\cos \theta_{k}\right)-f(\cos \theta)\right\} S_{k}[\theta] \\
= & \sum_{1 \leqq k \leqq n,\left|\theta-\theta_{k}\right| \leqq \delta}\left\{f\left(\cos \theta_{k}\right)-f(\cos \theta)\right\} S_{k}[\theta] \\
& +\sum_{1 \leqq k \leqq n,\left|\theta-\theta_{k}\right|>\delta}\left\{f\left(\cos \theta_{k}\right)-f(\cos \theta)\right\} S_{k}[\theta] .
\end{aligned}
$$

Then, by the use of (6) and (10), for sufficiently large $n$, we find that 


$$
\begin{aligned}
& |\Delta|<\epsilon \sum_{1 \leqq k \leqq n,\left|\theta-\theta_{k}\right| \leqq \delta}\left|S_{k}[\theta]\right|+2 M \sum_{1 \leqq k \leqq n,\left|\theta-\theta_{k}\right|>\delta}\left|S_{k}[\theta]\right| \\
& <\epsilon D+2 M O\left(\frac{1}{n}\right)<(D+1) \epsilon, \quad n>N \text {. }
\end{aligned}
$$

As in Rogosinki's theorem for Fourier series, the theorem may be easily extended so that convergence holds at any point $x(\neq \pm 1)$ of continuity of a bounded function $f(x)$, convergence being uniform in any closed interval of continuity (excluding $x= \pm 1$ ). In addition, $t_{n}$ may be replaced by $p \pi / 2(n+1)$ where $p$ is any fixed odd integer.

Since $L_{n}[f ;-t]=L_{n}[f ; t]$, the theorem would involve (for $\theta=0$ ) the convergence of $L_{n}[f ; t]$ to $f(\cos 0)$. This convergence does not hold for all continuous $f(x)$ because

$$
\lambda_{n} \equiv \sum_{k=1}^{n}\left|l_{k}(1)\right|=n
$$

and, according to H. Hahn [3], a necessary and sufficient condition for $L_{n}[f ; 0]$ to converge to $f(\cos 0)$ for all continuous $f(x)$ is that $\lambda_{n}$ be bounded for all $n$. We give an example (compare [4] and [5]) of a continuous function for which convergence does not occur at $x=1$.

Let $f_{n}(x)$ be defined for each $n(n=1,2, \cdots)$ as follows :

1) $f_{n}(x)=\left\{\begin{array}{cr}(-1)^{n-1}, & -1 \leqq x<x_{n}, \\ (-1)^{k}\left[1-\frac{2\left(x-x_{k+1}\right)}{x_{k}-x_{k+1}}\right], x_{k+1} \leqq x \leqq x_{k} ; k=1,2, \cdots, n-1, & \\ 1, & x_{1}<x \leqq 1 .\end{array}\right.$ Now,

$$
\begin{aligned}
L_{n}\left[f_{n} ; t\right] & =-\sum_{k=1}^{n}(-1)^{k} l_{k}[t] \\
& =\frac{2}{(n+1) \sin t} \sum_{k=1}^{n} \frac{\sin ^{2} k t \cos ^{2} k t}{\sin (k+1 / 2) t \sin (k-1 / 2) t} .
\end{aligned}
$$

Since

we have

$$
\frac{\sin k t}{\sin (k+1 / 2) t}>\frac{2}{3}, \quad k=1,2, \cdots, n,
$$

$$
\begin{aligned}
\left|L_{n}\left[f_{n} ; t\right]\right| & >\frac{4}{3(n+1) \sin t} \sum_{k=1}^{n} \cos ^{2} k t \\
& >\frac{2}{3(n+1) \sin t} \sum_{k=1}^{n}\left(1+\cos \theta_{k}\right)>\frac{4 n}{3 \pi}
\end{aligned}
$$


By the Weierstrass approximation theorem, we may approximate $f_{n}(x)$ by a polynomial $g_{n}(x)$ of degree $d(n)$ such that in $(-1,1)$,

$$
\left|g_{n}(x)\right| \leqq 3 / 2, \quad\left|L_{n}\left[g_{n} ; t\right]\right|>2 n / 3 \pi .
$$

This is possible, in view of (12), because

$$
\left|L_{n}\left[f_{n} ; t\right]-L_{n}\left[g_{n} ; t\right]\right|=\left|\sum_{k=1}^{n}\left[f_{n}\left(x_{k}\right)-g_{n}\left(x_{k}\right)\right] l_{k}[t]\right| \leqq \epsilon^{\prime}
$$

provided

$$
\left|f_{n}(x)-g_{n}(x)\right| \leqq\left(\epsilon^{\prime} / \sum_{k=1}^{n}\left|l_{k}[t]\right|\right), \quad-1 \leqq x \leqq 1 .
$$

Let

$$
g(x) \equiv \sum_{i=1}^{\infty} c_{i} g_{n_{i}}(x), \quad-1 \leqq x \leqq 1
$$

where $c_{1}=n_{1}=1$, and

$$
c_{i+1}=\min \left\{\frac{c_{i}}{4}, \frac{1}{T_{i}}\right\}, \quad T_{i} \equiv \sum_{k=1}^{n_{i}}\left|l_{k}^{(n i)}\left[t_{n_{i}}\right]\right|, \quad i=1,2, \cdots ;
$$

$n_{i}$ is the smallest integer satisfying the conditions

$$
\begin{aligned}
& \text { (a) } n_{i} \geqq d\left(n_{i-1}\right)+1 \\
& \text { (b) }\left|c_{i} L_{n_{i}}\left[g_{n_{i}} ; t_{n_{i}}\right]-8\right|>4^{i}, \quad i=2,3, \cdots .
\end{aligned}
$$

Condition (b) is possible because of (13). From (15), it follows that

$$
c_{i+1} \leqq 1 / 4^{i}, \quad i=0,1,2, \cdots,
$$

and the series for $g(x)$ converges uniformly so that $g(x)$ is continuous and $|g(x)| \leqq 2$ in $(-1,1)$. Let $m=n_{r}$ where $r(\neq 1)$ is a positive integer. Using (15) and (17), we have

$$
\begin{gathered}
\left|\sum_{i=r+1}^{\infty} c_{i} L_{m}\left[g_{n_{i}} ; t_{m}\right]\right| \leqq \sum_{i=r+1}^{\infty} c_{i} \frac{3}{2} \sum_{k=1}^{m}\left|l_{k}^{(m)}\left[t_{m}\right]\right| \leqq 2, \\
\left|\sum_{i=r}^{\infty} c_{i} g_{n_{i}}\left(\cos t_{m}\right)\right| \leqq 2 .
\end{gathered}
$$

If $w(x)$ is any polynomial of degree less than $m$ in $x=\cos \theta$, then $L_{m}[w ; \theta] \equiv w(x)$. Since 


$$
\begin{aligned}
\sum_{i=1}^{\infty} c_{i} L_{m}\left[g_{n_{i}} ; t_{m}\right] & \\
& =c_{r} L_{m}\left[g_{m} ; t_{m}\right]+\sum_{i=1}^{r-1} c_{i} L_{m}\left[g_{n_{i}} ; t_{m}\right]+\sum_{i=r+1}^{\infty} c_{i} L_{m}\left[g_{n_{i}} ; t_{m}\right] \\
& =c_{r} L_{m}\left[g_{m} ; t_{m}\right]+\sum_{i=1}^{r-1} c_{i} g_{n_{i}}\left(\cos t_{m}\right)+\sum_{i=r+1}^{\infty} c_{i} L_{m}\left[g_{n_{i}} ; t_{m}\right],
\end{aligned}
$$

we find from (16) and (18) that

$$
\begin{aligned}
&\left|L_{m}\left[g ; t_{m}\right]-g(1)\right| \\
&=\left|\left\{L_{m}\left[g ; t_{m}\right]-g\left(\cos t_{m}\right)\right\}+\left\{g\left(\cos t_{m}\right)-g(1)\right\}\right| \\
& \geqq\left|\sum_{i=1}^{\infty} c_{i} L_{m}\left[g_{n_{i}} ; t_{m}\right]-g\left(\cos t_{m}\right)\right|-4 \\
& \geqq\left|c_{r} L_{m}\left[g_{m} ; t_{m}\right]+\sum_{i=r+1}^{\infty} c_{i} L_{m}\left[g_{n_{i}} ; t_{m}\right]-\sum_{i=r}^{\infty} c_{i} g_{n_{i}}\left(\cos t_{m}\right)\right|-4 \\
&>4^{r}, \quad r=2,3, \ldots .
\end{aligned}
$$

\section{BIBLIOGRAPHY}

1. M. Webster, Note on certain Lagrange interpolation polynomials, Bull. Amer. Math. Soc. vol. 45 (1939) pp. 870-873.

2. G. Grünwald, On a convergence theorem for the Lagrange interpolation polynomials, Bull. Amer. Math. Soc. vol. 47 (1941) pp. 271-275.

3. H. Hahn, Über das Problem der Interpolation, Math. Zeit. vol. 1 (1918) pp. 115143.

4. P. Erdös and P. Turán, On interpolation. I, Ann. of Math. (2) vol. 38 (1937) pp. 142-155.

5. E. Feldheim, Quelques recherches sur l'interpolation de Lagrange et d'Hermite par la méthode du développement des fonctions fondamentales, Math. Zeit. vol. 44 (19381939) pp. 55-84.

Purdue University 